\title{
Utilization of Routine Health Information System and Associated Factors Among Health Professionals in Public Health Facilities in Dire Dawa, Eastern Ethiopia: a Cross-sectional Study
}

\section{Samuel Mekuria}

Dire Dawa Adminstration Health Bureau, Ministry of Health

Hassen Abdi Adem ( $\square$ hassenxiqqa32@gmail.com )

Haramaya University

Behailu Hawulte Ayele

Haramaya University

Ibsa Musa

Haramaya University

Daniel Berhanie

Haramaya University

\section{Research Article}

Keywords: Utilization, Health information system, Health professionals, Dire Dawa, Ethiopia

Posted Date: September 21st, 2021

DOI: https://doi.org/10.21203/rs.3.rs-907625/v1

License: (c) (i) This work is licensed under a Creative Commons Attribution 4.0 International License.

Read Full License 


\section{Abstract}

Background: Using reliable evidence from routine health information over time is an important aid to improve the health outcome, tackling disparities, enhancing efficiency, and encouraging innovation. In Ethiopia, the utilization of routine health data for improving the performance and quality of care was not well-studied in primary and secondary health facilities. This study assessed the level of routine health information utilization and associated factors among health professionals in public health facilities of Dire Dawa, eastern Ethiopia.

Method: An institution-based cross-sectional study was conducted among 378 randomly selected health professionals from June 10 to July 20, 2020. A self-administered pretested structured questionnaire was used to collect data from participants. Data were entered using EpiData version 3.1 and analyzed using Stata version 16.0. Descriptive statistics were used to characterize the participants and binary logistic regression analysis was conducted to identify factors associated with the utilization of routine health information. Adjusted Odds Ratio (AOR) with 95\% confidence interval was used to report association and significance was declared at $P$-value $<0.05$.

Results: Good utilization of routine health information among health professionals was $57.7 \%(95 \% \mathrm{Cl}$ : $52.6 \%, 62.6 \%$ ). Good organizational support ( $\mathrm{AOR}=3.91,95 \% \mathrm{Cl}: 2.01,7.61)$, the low perceived complexity of the reporting formats ( $A O R=2.20,95 \% \mathrm{Cl}: 1.23,3.97)$, good self-efficacy ( $A O R=2.52,95 \% \mathrm{Cl}: 1.25,5.10)$, and good decision making autonomy $(\mathrm{AOR}=3.97,95 \% \mathrm{Cl}: 2.12,7.43)$ were important factors associated with good utilization of routine health information.

Conclusion: Good utilization of routine health information among health professionals was low. Lack of self-confidence and empowerment of health professionals, the complexity of routine health information system format, and poor organizational support were significantly reducing the level of routine health information utilization. Therefore, improving the self-efficacy and decision-making capacity of health professionals through comprehensive training, empowerment and organizational support would be essential to increase the level of routine health information utilization.

\section{Introduction}

The routine health information system (RHIS) is a set of data regularly collected to meet predictable information needs primarily containing statistics on health services, epidemiological, administrative and financial data [1]. A well-functioning RHIS is essential to provide the information needed for health system management, for governance, accountability, planning, policy making, surveillance and quality improvement [2]. In Ethiopian context usually RHIS is equivalent to Health Management Information System (HMIS) which has been implemented since 2008 to provide core indicators used to improve the provision of healthcare services and health status of the population and used as a major source of information for monitoring and adjusting policy implementation [3, 4]. 
However, the use of information for evidence-informed decision making-particularly data produced by RHIS is still very weak in most low- and middle-income countries including Ethiopia [5, 6]. In these regions, utililization and effectiveness of RHIS in improving health system performance has been questioned due to multivariate factors/determinants [5]. Besides, the large amount of unreliable health data, poor human resources, and poor information technology infrastructure as well as poor utilization of the data for evidence based decisions cumbersome the effectiveness of the system [7]. Consequentially, too often, data are sat in reports, shelves, cabinets, databases and left unanalyzed to be sufficiently utilized for policy and program improvements [8].

Health professionals are expected to play a pivotal role in RHIS data analysis, interpretation, and utilization for operational, tactical, and strategic decision making. Despite these health professionals in health facilities are often over burdened by excessive reporting requirements from multiple and poorly coordinated subsystems that cannot deliver timely, accurate and complete data $[9,10]$. Besides, health professionals were the main source of poor data quality in general and incompleteness and incorrectness in particular mostly due to technical, behavioral/individual and environmental/organizational factors of information use $[11,12]$. According to the study conducted among health professionals in Addis Ababa, lack of appropriate inputs to the system, lack of RHIS data management skill, lack of incentives, lack of feedback, lack of technical support, poor attitude of health professionals, lack of management commitment, centralized decision making, and absence of information use culture were identified as an important factors for RHIS utilization [13].

In response to the aforementioned challenges, the ministry of health-Ethiopia has undertaken an extensive reform and redesign the national RHIS to respond to the deficiency of routine health data that limited the quality of care and decision making by managers at all levels $[3,4]$. Most importantly, the ministry has set information revolution as one of the four major priority agenda aimed to enhance behavioral/cultural transformation of health workers and digitalization of the health information system $[3,4]$. However, recent studies evidencing the current status of RHIS utilization is lacking $[2,5,14,15]$. Besides, majority of the previous studies limited to organizational and managerial levels to assess the utilization of RHIS $[2,5,14-19]$. Therefore, this study aimed to assess the level of RHIS/HMIS information utilization using objectively measured multivariate factors (technical, behavioral, and behavioral factors) among health professionals in public health facilities of Dire Dawa in Ethiopia.

\section{Methods}

\section{Study design and setting}

An institution-based cross-sectional study was conducted in Dire Dawa City Administration in Ethiopia from 10 June to 20 July 2020. Dire Dawa is located at 515 Kilometres, East of Addis Ababa, Capital of Ethiopia. Based on the 2007 Central Statistical Agency population census, the total population of Dire Dawa Administration was projected to be 342, 827 in 2019 [20]. According to the Dire Dawa 
Adminstration Health Bureau annual report of 2019, there were two hospitals, 15 health centers, and 34 health posts with a total of 1080 health workers [21].

\section{Population and sampling}

All health professionals in public health facilities in Dire Dawa were the source population. Health professionals in randomly selected public health facilities in Dire Dawa were the study population. Critically sick health professionals-and those who were on leave of any type or long-term training or education or field activities during the data collection period were excluded from the study.

The sample size was calculated with Epi Info version 7.1 considering the assumption for single (HMIS utilization) and double (for factors) population proportion formulas. Accordingly, maximum sample size of 379 was obtained using a single population proportion formula considering $78.5 \%$ proportion of good routine health information utilization [16], confidence level of $95 \%$, margin of error of $5 \%$, design effect of 2 , non-response of $10 \%$, and a total population of 1080 .

We applied a two-stage stratified sampling technique to select the study participants. First, public health facilities were stratified into hospitals and health centers, and then, one out of two hospitals and six out of 15 health centers were randomly selected. Then, the sample size was proportionally allocated to each selected facility based on the actual numbers of registered health professionals serving in the selected health facility during the last six months before the interview. We prepared a separate sampling frame for each health facility using their actual numbers of (permanently) hired health professionals in 2020 and recruited participants using a simple random sampling technique.

\section{Data collection tools and measurements}

Pretested-structured questionnaire adapted from PRISM framework assessment tool version 3.1 [7] and published literature $[13,14,16,19,22-28]$ were used to collect data from participants through a selfadministered interview conducted over a month. The questionnaire contains information on sociodemographic characteristics of the participants, technical factors, organizational factors, behavioral factors, and the level of utilization of routine health information (Supplementary Table 1).

Before starting analysis, internal consistencies of items were checked for each domain scale of dependent and independent variables using reliability analysis (Cronbach a). Accordingly, we checked for the internal consistency of each domain of PRISM framework tool for assessing organizational, technical and behavioral determinants of RHIS utilization and computed summary statistics, mean $\pm S D$, minimum and maximum scores, and standard error. We observed high internal consistency across all domains with the minimum in attitude toward RHIS items (Cronbach's $a=0.72$ ) and the maximum in self-efficacy items (Cronbach's a=0.97) (Supplementary Table 2).

Utilization of routine health information system (RHIS): The use of information for improving effectiveness and efficiency of healthcare services through better management at all levels [7]. Utilization of RHIS was measured using 13 five-points Likert Scale items each rated from ' 1 ' (strongly disagree) to ' 5 ' 
(strongly agree) and then, composite index score was computed from 13 items and the level of RHIS utilization was 'good' when the participants scored above the mean and 'poor' unless otherwise $[16,17$, 24].

Organizational support: It was measured using 11 five-points Likert Scale items each rated from ' 1 ' (strongly disagree) to ' 5 ' (strongly agree) and then, composite index score was computed from 11 items, and the level of perceived organizational support was 'good' when the participants scored "quartile 3 and above", 'fair' when scored between "quartile 3 and quartile 1" (excluding both) and 'poor' unless otherwise $[7,16]$.

Training: Considered 'yes' when the participants received a short-term education or orientation on health management information system and its related reporting tools and procedures according to the national health information system training manual on how to use data elements, indicators, and their definitions in the last two years and 'no' unless otherwise.

Training adequacy: It was measured using seven five-points Likert Scale items each rated from ' 1 ' (strongly disagree) to ' 5 ' (strongly agree) and then, composite index score was computed from seven items and the training adequacy was 'good' when the participant scored "quartile 3 and above", 'fair' when scored between "quartile 3 and quartile 1" (excluding both) and 'poor' otherwise [7, 16, 17].

Supervision: Considered 'yes' when the participant had taken health information system-specific or related supportive follow up aimed at enabling providers to perform RHIS properly through providing onjob training and technical support in the last six months and 'no' otherwise [7, 25].

Supervision quality: It was measured using five dichotomous items each coded ' 1 ' when the participants responded "right answer" and ' 0 ' when responded "the wrong answer" and then, composite index score was computed from five items and the level of supervision quality was 'good' when the participant scored "quartile 3 and above", 'fair' when scored between "quartile 3 and quartile 1" (excluding both) and 'poor' unless otherwise $[7,17]$.

Technical support: It was measured using seven five-points Likert Scale items each rated from ' 1 ' (strongly disagree) to ' 5 ' (strongly agree) and composite index score was computed from seven items, and the technical support was 'good' when the participant scored "quartile 3 and above", 'fair' when scored between "quartile 3 and quartile 1" (excluding both) and 'poor' otherwise [7, 17].

The perceived complexity of RHIS formats: It was measured using four dichotomous (yes/no) questions each coded ' 1 ' when the participant responded 'yes' (i.e. complex) and ' 0 ' when responded 'no' (i.e. not complex) and then, composite index score was computed from four items and level of the perceived complexity of RHIS formats was "not complex" when the participant scored "quartile 1 and below", "fairly complex" when scored between "quartile 1 and quartile 3" (excluding both) and 'complex' when scored between "quartile 3 and above " $[7,17]$. 
Self-efficacy: It was measured using seven 10-points Likert Scale items each rated from '0' (no competency) to '10' (best competency) and composite index score was computed from seven items and the self-efficacy was 'good' when the participant scored "quartile 3 and above", 'fair' when scored between "quartile 3 and quartile 1" (excluding both) and 'poor' unless otherwise $[7,17]$.

Attitude toward RHIS: It was measured using six five-point Likert Scale items each rated from '1' (strongly disagree) to ' 5 ' (strongly agree) and composite index score was computed from six items, and health professional's attitude, was 'good' when participant scored "quartile 3 and above", 'fair' when between "quartile 3 and quartile 1" (excluding both) and 'poor' unless otherwise [7, 14, 16].

Decision-making autonomy: It was measured using six five-points Likert Scale items each rated from '1' (strongly disagree) to ' 5 ' (strongly agree) and composite index score was computed from six items and decision-making autonomy was 'good' when the participants scored "quartile 3 and above", 'fair' when scored between "quartile 3 and quartile 1" (excluding both) and 'poor' unless otherwise [7, 16, 17, 24].

\section{Data quality control}

To maintain the data quality, standard questionnaires adapted from validated scales and published literature were contextualized to the study purpose and setting. Six trained nurses collected the data, and two experts holding BSc degree in Public Health was supervised the overall data collection process with the principal investigator. We also pretested a questionnaire on 19 health professionals ( $5 \%$ of the total sample) to check its validity in Melka Jabdu Health Center (a non-selected health facility in Dire Dawa, Ethiopia). Epi-Data was used for data entry to minimize the potential errors that could occur during entry. During data collection, strict supervision of data collectors and validation of collected data was carried out by supervisors and investigators.

\section{Statistical analysis}

After checking for completeness, data were entered using EpiData version 3.1 and analyzed using Stata version 16.0. Descriptive statistics such as frequency, measures of central tendency, and dispersion were used to characterize the participants. Bivariable and multivariable binary logistic regression analyses were conducted to identify factors associated with good utilization of routine health information. Independent variables with P-value $<0.25$ during our bivariable analysis were considered in our multivariable analysis model. The overall model adeaucy was confirmed using Hosmer and Lemeshow goodness of fit test at P-value $>0.05$. We rulled out and confirmed the absence of numerical errors and multicolliniearity in the model (each predictor has standardized resiedul of less than absolute value of three and cook's distance of less than absolute value of one and their standard errors of each cofficient $(\beta)$ was less than two). Adjusted Odds Ratio(AOR) with a 95\% confidence interval was used to report association and significance declared at $P$-value $<0.05$.

\section{Results}




\section{Characteristics of participants}

A total of $378(99.7 \%)$ health professionals were involved in the study. Three hundred fifty-six $(94.2 \%)$ participants were urban residents and half $(50.8 \%)$ of the participants were female. The mean age $( \pm S D)$ of the participants was $31.5 \pm 6.6$ years, and $263(69.6 \%)$ of the participants were in the age group of 2635 years. The majority $(79.9 \%)$ of participants were Bachelor of Science degree holders, more than half (57.9\%) were working in Health Center, and a quarter (25.1\%) of participants was a member of the facility's Performance Monitoring Team (PMT). Around half (51.3\%) of participants had been serving in at least two health facilities, and the median duration of work experience of the participants was four years (Quartile 1=2, Quartile 3=8) (Table 1).

\section{Organizational, technical and individual factors}

From a total of 378 health professionals, perceived organizational support was good for $25.9 \%$ of the participants. Around a quarter (27.8) of participants received RHIS training in the the last six months and the perceived training adequacy was good for $28.6 \%$ of the trained participants. About $63.2 \%$ of the participants had received RHIS supervision in the last six months (prior to start study) from the higherlevel institution(s) and the perceived supervision quality was good for $40.7 \%$ of the supervised participants. Nearly three out of ten (29.4\%) participants had good perceived technical support. A quarter $(25.1 \%)$ and one-third (29.9\%) of participants had good self-efficacy and good decision-making autonomy, respectively (Table 2 ).

\section{Utilization of routine health information system}

This study identified, the level of good routine health information utilization was $57.7 \%$ (95\% Cl: $52.6 \%$, $62.6 \%$ ) among health professionals in public health facilities of Dire Dawa, eastern Ethiopia

\section{Factors associated with utilization of routine health information system}

The bivariable analysis showed that sex, type of facility, being a PMT member, organizational support, supervision, technical support, perceived complexity of RHIS format, health professional attitude toward RHIS, self-efficacy, and decision making autonomy were significantly associated with the good level of routine health information system utilization at P-value $<0.05$. Predictors with $P$-value $<0.25$ in the bivariable analysis were included in our multivariable analysis model. Accordingly, good (AOR=3.91, 95\% Cl: $2.01,7.61)$ and fair (AOR=1.94, 95\% Cl: 1.14, 3.30) levels of organizational support, good self-efficacy (AOR=2.52, 95\% Cl: 1.25, 5.10), and good decision-making autonomy (AOR=3.97, 95\% Cl: 2.12, 7.43) among health professionals and the percieved simplicity (non-complexicity) of RHIS formats (AOR=2.20, $95 \% \mathrm{Cl}: 1.23,3.97)$ were positively associated with the good level of routine health information utilization (Table 3).

\section{Discussion}


This study investigated the level of routine health information utilization and associated factors among health professional in public health facilities in Dire Dawa, eastern Ethiopia. We found that about half (57.7\%) of health professionals had good level of routine health information utilization. The level of organizational support, the perceived complexity of RHIS formats, self-confidence and decision making ability among health professionals were identified independent predictors of the level of routine health information utilization.

Accordingly, this study revealed that around one out of two (57.7\%) health professionals had the good level of routine health information system utilization which implies substantial proportion of health professionals are utilizing routine health information system. This finding is higher than the studies conducted in Harar, eastern Ethiopia (30.6\%) [29], Jimma, southwest Ethiopia (32.9\%) [28] and Gojjam, northern Ethiopia (45.8\%) [24]. In addition, it is higher than study conducted in eastern Ethiopia (54.4\%) [12] and the national studies conducted in Ethiopia (48\%) [30] and Cote d'Ivoire (38\%) [8]. However, this finding was lower than the study conducted in North Gondar, northwest Ethiopia (78.5\%) [16]. The possible explanations for the higher good level of routine health information utilization in this study might be due to the better organizational support, non complixicity of RHIS formats and better selfconfidence and decision-making autonomies of health professionals seen in the present study. In addition, recently the government special emphasis to the utilization of routine health information for evidence-based decision making and improvement of health professionals' culture of information use might be other possible explanation [31].

Moreover, few previous studies were done in the settings where there were no full structure and assigned personnel on RHIS/HMIS program (health post and health centers) that might decreased the good level of RHIS utilization $[28,32,33$ ] while other previous studies with the higher good routine health information utilization might due to the methodologies variation (inclusion criteria) used to select the participants. For instance, in North Gondar study, participants were the most influential PMT members of the health facilities like the HMIS/HIS officers/HIT professionals, facilities head and departments/case team leaders [16].

In this study, the better level of organization support was significantly increased with the good level of routine health information utilization. Routine health information utilization was fourfolds higher among health professionals who had good and fair organizational support from higher/above bodies compared to health professionals who had poor organizational support. This finding was somewhat similar with studies conducted in Western Amhara and East Gojjam Zone, northwest Ethiopia that showed organizational factors such as access to training, supervision, computer, HMIS formats and guideline was a significant predictor of the good routine health information utilization [24, 32].

In addition, this findings was supported by study conducted in northern Ethiopia that indicated good culture of information use, supervision, governance, planning and feedback were increased the utilization of routine health information [16]. Moreover, this could be due to presence of program-specific regular supportive supervision and feedback provision sytem seen in our study setting and recently, the 
government special emphasis to utilization of RHIS for evidence-based decision making and improvement of health professionals' culture of information use might be raised the level of organizational support and RHIS utilization [31].

According to the WHO measure evaluation, self-efficay is one of determinants of RHIS utilization [34]. Health professionals' self-efficacy came from knowledge and understanding about HMIS that in turn lead to good utilization of health information for decision-making. In this study, good self-efficacy was statistically significant predictor of routine health information utilization. The RHIS utilization was around three-fold higher among health professionals who had good self-efficacy compared to those who had poor self-efficacy. This finding was similar with the study conducted in Hadiya Zone, southern Ethiopia; the RHIS utilization was three-folds higher among health workers who had the good self-efficacy enough to perform HMIS activities compared to their counterpart [19]. The finding was also supported by study done in southern Ethiopia; health information data quality was higher among health workers having good self-efficacy enough to perform HMIS activities compared to their counterpart [26, 27]. Moreover, this was supported by studies done in Ethiopia and Uganda; health workers who were confident enough to perform HMIS activities were more likely to use routine health information than their counterpart $[19,27,35]$.

Besides, the possible explanation, the RHIS utilization was influenced by competence of the people to perform HMIS tasks and in this study, there were higher confidence level for computing trends, using data for identifying gaps and setting targets, using data for various types of decisions and providing feedback whereas the lower competence level observed in explaining the trend obtained from data, explaining findings, and culture of information use. These shows unawareness of a gap between their perceived and actual competence in performing a task. In this study, routine health information utilization was threefolds higher when health professionals had good decision-making power compared to their counterpart. This finding was similar with studies done in East Wollega Zone, western Ethiopia [17] and eastern Ethiopia [14].

As the strength, this study attempted to show the level and predictors of routine health information utilization among health professionals. In addition, the study used standard validated instrument, a PRISM framework version 3.1 tool for data collection. Moreover, it can be generalized to public health facilities in urban and rural settings. In addition, the study was not free from limitations such as inability to include qualitative method to explore health professionals' culture of information use and other organizational factors. Moreover, the cross-sectional study design might have prevented the work from showing temporal relationship. Besides, the study was not able to include health professionals in private institutions.

\section{Conclusion}

This study concluded that about half of health professionals in public health facilities in Dire Dawa had practiced the good routine health information utilization. In this study, organizational support, the perceived complexity of RHIS formats, level of self-efficacy and decision making autonomy were found to 
be significant predictor of the good level of routine health data utilization. Therefore, provision of comprehensive health information system training, strengthening organization support and empowering decision making capacity of healthcare providers in public health facilities would be essential. Furthermore, further research is suggested for assessing health workers' culture of health information use at the lower health facilities where data are generated.

\section{Abbreviations}

AOR: Adjusted Odds Ratio, HMIS: Health Management Information System, PMT: Performance Monitoring Team, PRISM: Performance of Routine Information System Management, RHIS: Routine Health Information System, WHO: World Health Organization

\section{Declarations}

\section{Competing interests}

The authors declare that there is no conflict of interests or competing interests with respect to the research, authorship and/or publication of this article.

\section{Ethical approval and informed consent}

The study was conducted in accordance with the Helsinki Declaration of researches involving human subjects [36]. Institutional Health Research Ethical Review Committee of the College of Health and Medical Sciences, Haramaya University approved the protocol of the study (Ref.no: IHRERC/142/2020). Formal permission was obtained from Dire Dawa Regional Health Bureau and selected health facilities. Informed, voluntary, written and signed consent was obtained from each participant after explaining the purpose and benefits of the study. Participants filled questionnaire in a separate area after informed the collected information would be kept confidential and not shared.

\section{Funding}

The study was funded by Haramaya University as part of master of public health (MPH) study to SM.The funder has no role in the design, execution, analysis or decision for publication.

\section{Availability of data and materials}

The data of this study are presented in the main manuscript. Any additional files (data) that support the findings is available from the correspondence author on reasonable request.

\section{Authors' contributions}

SM, HAA, BHA, IM and DBE participated in the conception of the idea, development of the proposal, data collection, and analysis, and wrote up the draft results. HAA and BHA reanalyzed the data, drafted, edited 
and revised the drafted and revised manuscript. All authors read and approved the final manuscript.

Acknowledgments

We acknowledged the participants and supervisors for their valuable contribution and cooperation. The authors thank Haramaya University for providing the opportunity to conduct the study. We also appreciated Dire Dawa Adminstration Health Bureau for providing the background information and medical records of the study setting.

Consent for publication

Not applicable

\section{References}

1. Ahanhanzo YG, Ouendo E-M, Kpoze`houen A, Leve^que A, Makoutode M, Dramaix-Wilmet MI: Data quality assessment in the routine health information system: an application of the Lot Quality Assurance Sampling in Benin. Health Policy and Planning 2015, 30:837-843.

2. Leon N, Balakrishna Y, Hohlfeld A, Odendaal W, Schmidt B-M, Zweigentha V, Anstey WJ, Daniels K: Routine Health Information System (RHIS) improvements for strengthened health system management. Cochrane Database of Systematic Reviews 2020, 8(CD012012).

3. FMOH: Health Sector Transformation Plan. In.; 2015.

4. FMOH: National Health Information System Road Map. In.; 2012.

5. Lemma S, Janson A, Persson L-k, Wickremasinghe D, Ka“llestål C: Improving quality and use of routine health information system data in low- and middle-income countries. A scoping review. PLOS ONE 2020, 15(10):e0239683.

6. WHO: Routine health information systems: a curriculum on basic concepts and practice. Measure Evaluation. Facilitators' guide In.; 2017.

7. Aqil A, Lippeveld T, Hozumi D: PRISM framework: a paradigm shift for designing, strengthening and evaluating routine health information systems. Health Policy Plan 2009, 24(10):217-228.

8. Nutley T, Gnassou L, Traore M, Bosso AE, Mullen S: Moving data off the shelf and into action: Anintervention to improve data-informed decision making in Côte d'Ivoire. Global Health Action 2014, 7(1):25035.

9. WHO: Standards for country health information systems /health metrics network. In. Geneva: World Health Organization; 2008.

10. FMOH: Health Sector Development Programme IV: 2010/11-2014/15 In.; 2010.

11. WHO/HMN: Framework and Standards for Country Health Information Systems. 2010.

12. Teklegiorgis $\mathrm{K}$, Tadesse $\mathrm{K}$, Mirutse G, Terefe W: Level of data quality from Health Management Information Systems in a resources limited setting and its associated factors, eastern Ethiopia. South African Journal of Information Management 2016, 17(1):a612. 
13. Mengistu A: Assessment of magnitude and factors affecting health information system useinprivatate and public health facilities in Addis Ababa. Masters thesis, Addis Ababa University, Ethiopia. 2009.

14. Teklemariam E, Mekonnen Y, Assefa K: Utilization of HMIS Data and Its Determinants at Health Facilities in East Wollega Zone, Oromia Regional State, Ethiopia: A Health Facility Based CrossSectional Study. 2018.

15. Odei-Lartey EO, Prah RKD, Anane EA, Danwonno H, Gyaase S, Oppong FB, Afenyadu G, Asante KP: Utilization of the national cluster of district health information system for health service decisionmaking at the district, sub-district and community levels in selected districts of the Brong Ahafo region in Ghana. BMC Health Services Research 2020, 20(1):514.

16. Dagnew E, Woreta SA, Shiferaw AM: Routine health information utilization and associated factors among health care professionals working at public health institution in North Gondar, Northwest Ethiopia. BMC Health Services Research 2018, 18(1):685.

17. Dufera FN, Lamenew W, Demissie DB, Guda A: Assessment of Behavioral and Organizational Determinants of HMIS Performance in Beghi, District West Wollega, Oromia, Ethiopia. Journal of Health, Medicine and Nursing 2018, 46.

18. Endriyas M, Alano A, Mekonnen E, Ayele S, Kelaye T, Shiferaw M, Misganaw T, Samuel T, Hailemariam T, Hailu S: Understanding performance data: health management information system data accuracy in Southern Nations Nationalities and People's Region, Ethiopia. BMC Health Services Research 2019, 19:175.

19. Wude $H$, Woldie $M$, Melese $D$, Lolaso $T$, Balcha B: Utilization of routine health information and associated factors among health workers in Hadiya Zone, Southern Ethiopia. PLOS ONE 2020, 15(5):e0233092.

20. CSA: Population Census of Ethiopia. 2007.

21. DDRHB: Dire Dawa Regional Health Bureau Annual Report of 2019 (Unpublished Data). In.; 2020.

22. Tadesse K, Gebeyoh E, Tadesse G: Assessment of health management information system implementation in Ayder referral hospital, Mekelle, Ethiopia. International Journal of Intelligent Information Systems 2014, 3(4):34.

23. Stephanie MTL, Edwards M, Kola TA, AJPaME O: Guidelines for Data Management Standards in Routine Health Information Systems. In.; 2015.

24. Shiferaw AM, Zegeye DT, Assefa S, Yenit MK: Routine health information system utilization and factors associated there of among health workers at government health institutions in East Gojjam Zone, Northwest Ethiopia. BMC Medical Informatics and Decision Making 2017, 17:116.

25. Asemahagn MA: Determinants of routine health informationutilization at primary healthcare facilities in Amhara, Ethiopia. Cogent Medicine 2017, 4:1387971.

26. Abera Eea: Utilization of Health Management Information System and Associated Factors in Hadiya Zone Health Centers, Southern Ethiopia. In: https://wwwresearchgatenet/publication/307626513. vol. 1; 2016. 
27. Belay H, Azim T, Kaddahun H: Assessment of Health Management Information System (HMIS) Performance in SNNPR, Ethiopia. Measure Evaluation. 2013.

28. Abajebel S, Jira C, Beyene W: Utilization of health information system at district level in Jimma zone Oromia regional state, South West Ethiopia. Ethiopian Journal of Health Sciences 2011, 21(3).

29. Mahtsentu: Assessment of Health Management Information System in Harari Regional State, Ethiopia. 2010.

30. WHO: Framework and Standards for Country Health Information Systems. In.; 2012.

31. Hotchkiss D, Aqil A, Mukooyo TL: Evalution of performance routine information system management (PRISM) framework evidence from Uganda. BioMed central 2010.

32. Asemahagn MA: Determinants of routine health information utilization at primary healthcare facilities in Western Amhara, Ethiopia. Cogent Medicine 2017, 4:1387971.

33. Shiferaw K, Musa A: Assessment of utilization of long acting reversible contraceptive and associated factors among women of reproductive age in Harar City, Ethiopia. Pan African Medical Journal 2017, 28(1):222.

34. Aqil A: PRISM framework: a paradigm shift for designing, strengthening and evaluating routine health information systems. 2009.

35. Hotchkiss D, Aqil A, Mukooyo E, Lippeveld T: Evaluation of the Performance of Routine Information System Management (PRISM) framework: evidence from Uganda. BMC Health Services Research 2010, 10:188.

36. CIOMS, WHO: International Ethical Guidelines for Biomedical Research Involving Human Subjects. 1st edition; 2008.

\section{Tables}


Table 1

Sociodemographic characteristics of health professionals in public health facilities in Dire Dawa, eastern Ethiopia, 2020 $(n=378)$

\begin{tabular}{|c|c|c|}
\hline Characteristic & Frequency & Percent \\
\hline \multicolumn{3}{|l|}{ Sex } \\
\hline Female & 192 & 50.8 \\
\hline Male & 186 & 49.2 \\
\hline \multicolumn{3}{|l|}{ Age (in years) } \\
\hline$\leq 25$ & 48 & 12.7 \\
\hline $26-35$ & 263 & 69.6 \\
\hline$\geq 36$ & 67 & 17.7 \\
\hline \multicolumn{3}{|l|}{ Current marital status } \\
\hline Currently married & 238 & 63.0 \\
\hline Currently Unmarried ${ }^{\star a}$ & 140 & 37.0 \\
\hline \multicolumn{3}{|l|}{ Education level } \\
\hline Diploma/Level & 55 & 14.6 \\
\hline Bachelor of Science degree & 302 & 79.9 \\
\hline Master of Science degree & 21 & 5.6 \\
\hline \multicolumn{3}{|l|}{ Religion } \\
\hline Orthodox & 220 & 58.2 \\
\hline Muslim & 91 & 24.1 \\
\hline Protestant & 56 & 14.8 \\
\hline Catholic & 11 & 2.9 \\
\hline \multicolumn{3}{|l|}{ Profession } \\
\hline Public Health & 37 & 9.8 \\
\hline Nurse & 176 & 46.6 \\
\hline Midwifery & 40 & 10.6 \\
\hline Laboratory & 26 & 6.9 \\
\hline Pharmacy & 31 & 8.2 \\
\hline Physician & 39 & 10.3 \\
\hline
\end{tabular}




\begin{tabular}{|c|c|c|}
\hline Other ${ }^{\star b}$ & 29 & 7.7 \\
\hline \multicolumn{3}{|l|}{ Type of health facility } \\
\hline Health Center & 219 & 57.9 \\
\hline Hospital & 159 & 42.1 \\
\hline \multicolumn{3}{|c|}{ Current working unit/ward } \\
\hline ART & 24 & 6.3 \\
\hline $\mathrm{MCH}$ & 90 & 23.8 \\
\hline OPD & 184 & 48.7 \\
\hline Pharmacy & 28 & 7.4 \\
\hline Laboratory & 25 & 6.6 \\
\hline Other ${ }^{*} \mathrm{c}$ & 27 & 7.1 \\
\hline \multicolumn{3}{|l|}{ Member of PMT } \\
\hline Yes & 95 & 25.1 \\
\hline No & 283 & 74.9 \\
\hline \multicolumn{3}{|c|}{ Working experience (in years) } \\
\hline$<5$ & 217 & 57.4 \\
\hline $5-10$ & 127 & 33.6 \\
\hline$>10$ & 34 & 9.0 \\
\hline \multicolumn{3}{|c|}{ Monthly Salary (in Ethiopian birrs) } \\
\hline$\leq 5087$ & 94 & 24.9 \\
\hline $5088-7999$ & 175 & 46.3 \\
\hline$\geq 8000$ & 109 & 28.8 \\
\hline
\end{tabular}

Note: RHIS=Routine Health Information System, PMT: Performance Monitoring Team, OPD: Outpatient Department, MCH: Maternal and child health, ART: Ante-retroviral Therapy a=Single/divorced/widowed, $b=$ radiographer, social worker/counselor and physiotherapist; $c=$ radiology unit, eye clinic, counseling unt and abortion 
Table 2

Organizational, technical, and behavioral determinants of utilization of routine health information system among health professionals in public health facilities in Dire Dawa, eastern Ethiopia, $2020(n=378)$

\begin{tabular}{|c|c|c|}
\hline Characteristic & Frequency & Percent \\
\hline \multicolumn{3}{|l|}{ Organization support } \\
\hline Poor & 122 & 32.3 \\
\hline Fair & 158 & 41.8 \\
\hline Good & 98 & 25.9 \\
\hline \multicolumn{3}{|l|}{ Training } \\
\hline Yes & 105 & 27.8 \\
\hline No & 273 & 72.2 \\
\hline \multicolumn{3}{|c|}{ Training adequacy $(n=105)$} \\
\hline Poor & 30 & 28.6 \\
\hline Fair & 45 & 42.9 \\
\hline Good & 30 & 28.6 \\
\hline \multicolumn{3}{|l|}{ Supervision } \\
\hline Yes & 239 & 63.2 \\
\hline No & 139 & 36.8 \\
\hline \multicolumn{3}{|c|}{ Supervision quality ( $\mathrm{n}=239$ ) } \\
\hline Poor & 11 & 4.6 \\
\hline Fair & 74 & 31.0 \\
\hline Good & 154 & 64.4 \\
\hline \multicolumn{3}{|l|}{ Technical support } \\
\hline Poor & 123 & 32.5 \\
\hline Fair & 144 & 38.1 \\
\hline Good & 111 & 29.4 \\
\hline \multicolumn{3}{|c|}{ Percieved complexity of RHIS formats } \\
\hline Not complex & 147 & 38.9 \\
\hline Fairly complex & 134 & 35.4 \\
\hline Complex & 97 & 25.7 \\
\hline
\end{tabular}




\begin{tabular}{|lll|} 
Self-efficacy & & \\
Poor & 98 & 25.9 \\
Fair & 185 & 48.9 \\
Good & 95 & 25.1 \\
Attitude toward RHIS & & \\
Poor & 136 & 36.0 \\
Fair & 131 & 34.7 \\
Good & 111 & 29.4 \\
Decision making autonomy & & \\
Poor & 175 & 46.3 \\
Fair & 90 & 23.8 \\
Good & 113 & 29.9 \\
\hline
\end{tabular}

Notes: RHIS=Routine Health Information System 
Table 3

Factors associated with utilization of routine health information system among health professionals in public health facilities in Dire Dawa, eastern Ethiopia, 2020 ( $n=378)$

\begin{tabular}{|c|c|c|c|c|}
\hline \multirow[b]{2}{*}{ Characteristic } & \multicolumn{2}{|c|}{ Utilization of RHIS } & \multirow[t]{2}{*}{$\operatorname{cOR}(95 \% \mathrm{Cl})$} & \multirow[t]{2}{*}{ aOR $(95 \% \mathrm{Cl})$} \\
\hline & Good, n (\%) & Poor, n (\%) & & \\
\hline \multicolumn{5}{|l|}{ Sex } \\
\hline Female & $120(62.5)$ & $72(37.5)$ & $1.497(0.99,2.26)^{*}$ & $1.45(0.91,2.32)$ \\
\hline Male & $98(52.7)$ & $88(45.4)$ & 1.00 & 1.00 \\
\hline \multicolumn{5}{|l|}{ Profession } \\
\hline Public Health & $25(67.6)$ & $12(32.4)$ & $1.98(0.78,5.03)$ & $1.11(0.39,3.58)$ \\
\hline Nurse & $107(60.8)$ & $69(39.2)$ & $1.47(0.73,2.96)$ & $0.98(0.41,2.23)$ \\
\hline Midwifery & $24(60.0)$ & $16(40.0)$ & $1.43(0.58,3.48)$ & $0.97(0.33,2.86)$ \\
\hline Laboratory & $12(46.2)$ & $14(53.8)$ & $0.81(0.30,2.20)$ & $1.02(0.33,3.18)$ \\
\hline Pharmacy & 13(41.9) & $18(58.1)$ & $0.69(0.27,1.78)$ & $0.43(0.14,1.35)$ \\
\hline Other & $17(58.6)$ & $12(41.4)$ & $1.35(0.51,3.60)$ & $1.27(0.41,3.97)$ \\
\hline Physician & $20(51.3)$ & 19(48.7) & 1.00 & 1.00 \\
\hline \multicolumn{5}{|l|}{ Type of health facility } \\
\hline Health Center & $139(63.5)$ & $80(36.5)$ & $1.76(1.16,2.67)^{\star \star}$ & $1.14(0.74,1.85)$ \\
\hline Hospital & $79(49.7)$ & $80(50.3)$ & 1.00 & 1.00 \\
\hline \multicolumn{5}{|l|}{ PMT member } \\
\hline Yes & $66(69.5)$ & $29(30.5)$ & $1.96(1.20,3.22)^{\star \star}$ & $1.01(0.54,1.83)$ \\
\hline No & $152(53.7)$ & $131(46.3)$ & 1.00 & 1.00 \\
\hline \multicolumn{5}{|l|}{ Organization support } \\
\hline Poor & $46(37.7)$ & $76(47.50)$ & 1.00 & 1.00 \\
\hline Fair & $94(59.5)$ & $64(40.5)$ & $2.43(1.50,3.94)^{\star \star \star}$ & $1.94(1.14,3.30)^{*}$ \\
\hline Good & 78(35.78) & $20(12.50)$ & $6.44(3.49,11.89)^{\star \star \star}$ & $3.91(2.01,7.61)^{\star \star \star}$ \\
\hline \multicolumn{5}{|l|}{ Supervision } \\
\hline Yes & $148(61.9)$ & $91(38.1)$ & $1.60(1.05,2.45)^{*}$ & $1.05(0.52,1.50)$ \\
\hline No & $70(50.7)$ & $69(49.3)$ & 1.00 & 1.00 \\
\hline
\end{tabular}




\begin{tabular}{|c|c|c|c|c|}
\hline Poor & $54(43.9)$ & $69(56.1)$ & 1.00 & 1.00 \\
\hline Fair & $85(59.0)$ & $59(41.0)$ & $1.84(1.13,2.99)^{*}$ & $1.36(0.78,2.38)$ \\
\hline Good & 79(71.2) & $32(28.8)$ & $3.16(1.83,5.43)^{\star \star \star}$ & $0.84(0.42,1.69)$ \\
\hline \multicolumn{5}{|c|}{ Complexity of RHIS formats } \\
\hline Complex & $41(42.3)$ & $56(57.7)$ & 1.00 & 1.00 \\
\hline Fairly complex & $75(56.0)$ & $59(25.0)$ & $1.74(1.02,2.94)^{*}$ & $1.17(0.64,2.12)$ \\
\hline Not complex & $102(69.4)$ & $45(30.6)$ & $3.10(1.82,5.28)^{\star \star \star}$ & $2.20(1.23,3.97)^{\star \star}$ \\
\hline \multicolumn{5}{|l|}{ Self-efficacy } \\
\hline Poor & $37(37.8)$ & $61(62.2)$ & 1.00 & 1.00 \\
\hline Fair & $108(58.4)$ & $77(41.6)$ & $2.31(1.40,3.82)^{\star *}$ & $1.56(0.89,2.74)$ \\
\hline Good & 73(78.6) & $22(21.4)$ & $5.47(2.92,10.25)^{\star \star \star}$ & $2.52(1.25,5.10)^{*}$ \\
\hline \multicolumn{5}{|l|}{ Attitude toward RHIS } \\
\hline Poor & $61(44.9)$ & $75(55.1)$ & 1.00 & 1.00 \\
\hline Fair & 73(55.7) & $58(44.3)$ & $1.55(0.96,2.51)$ & $1.17(0.65,2.08)$ \\
\hline Good & $84(75.7)$ & $27(24.3)$ & $3.83(2.21,6.63)^{\star \star \star}$ & $1.59(0.81,3.12)$ \\
\hline \multicolumn{5}{|c|}{ Decision-making autonomy } \\
\hline Poor & $77(44.0)$ & $98(56.0)$ & 1.00 & 1.00 \\
\hline Fair & $47(52.2)$ & $43(47.8)$ & $1.39(0.84,2.32)$ & $1.03(0.59,1.82)$ \\
\hline Good & $94(83.2)$ & 19(16.8) & $6.30(3.54,11.21)^{\star \star \star}$ & $3.97(2.12,7.43)^{\star \star \star}$ \\
\hline
\end{tabular}

Notes: Significant at $\mathrm{P}<0.05=*$, at $\mathrm{P}<0.01=* \star, \mathrm{P}<0.001=* \star * ;$ RHIS=Routine Health Information System, PMT: Performance Monitoring Team

\section{Supplementary Files}

This is a list of supplementary files associated with this preprint. Click to download.

- SuplementaryTable1.doc

- SuplementaryTable2.doc 